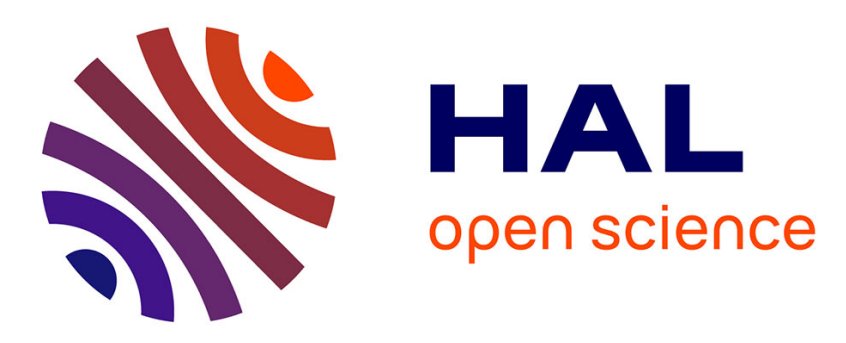

\title{
Fabrication of surface acoustic wave wireless pressure sensor
}

\author{
D. Hermelin, W. Daniau, S. Ballandras, B. Belgacem
}

\section{To cite this version:}

D. Hermelin, W. Daniau, S. Ballandras, B. Belgacem. Fabrication of surface acoustic wave wireless pressure sensor. 2009 IEEE International Frequency Control Symposium Joint with the 22nd European Frequency and Time forum, Apr 2009, Besançon, France. pp.96-99, 10.1109/FREQ.2009.5168149 . hal-00485757

\section{HAL Id: hal-00485757 \\ https://hal.science/hal-00485757}

Submitted on 12 May 2021

HAL is a multi-disciplinary open access archive for the deposit and dissemination of scientific research documents, whether they are published or not. The documents may come from teaching and research institutions in France or abroad, or from public or private research centers.
L'archive ouverte pluridisciplinaire HAL, est destinée au dépôt et à la diffusion de documents scientifiques de niveau recherche, publiés ou non, émanant des établissements d'enseignement et de recherche français ou étrangers, des laboratoires publics ou privés.

\section{(c)(1)}

Distributed under a Creative Commons Attribution| 4.0 International License 


\section{Fabrication of Surface Acoustic Wave wireless pressure sensor}

\author{
D. Hermelin, W. Daniaux, S. Ballandras \\ FEMTO-ST, UMR 6174 \\ Dept Temps-Fréquence \\ 26 Chemin de l'Epitaphe \\ 25000 Besançon, France
}

\author{
B. Belgacem \\ SENSeOR SAS \\ TEMIS Innovation Building \\ 18 Rue Alain Savary \\ 25000 Besançon
}

\begin{abstract}
This paper deals with walk-through allowing to explain the electrical response of a surface acoustic wave (SAW) resonator under inhomogeneous stress. Act of pressure on a circular membrane where is put the resonator induces inhomogeneous stress and changes the resonance frequency and the quality factor of the resonator. This paper describes a developped model based on P-matrix approach allowing to explain the behavior of these two parameters.
\end{abstract}

Keywords-component; SAW resonator, inhomogeneous stress distribution, quality factor, electrical impedance, pressure sensor

\section{INTRODUCTION}

This paper deals with modeling of surface acoustic wave resonator under inhomogeneous stress distribution. In the first part, sensor manufacturing and clean-room process are detailed. In the second part, a model of inhomogeneous stress distribution in the resonator is developped. Indeed, the sensor consists of a SAW resonator put in the center of a thinned circular clamped plate which sees its stress varying with the pressure and the position. The model allows to assess resonance frequency variation and Q-factor behavior when pressure is applied on the membrane. Moreover, this model is general but is particularly detailed in the the developped sensor. Finally, theoretical analysis and experimental datas are compared.

\section{SENSOR MANUFACTURING}

The figure 1 is a schematic view of the fabricated sensor. The sensor is composed of an AT-cut quartz wafer (1) and a silicon wafer (2). A resonator composed of IDTs (4) and Bragg mirrors (5) is placed on the center of the free quartz membrane. The diameter of the membrane is $8 \mathrm{~mm}$ and the total length of the resonator is about $3 \mathrm{~mm}$. The cavity in silicon (3) is performed using Deep Reactive Ion Etching (DRIE). The depth of the cavity is about $20 \mu \mathrm{m}$. The silicon wafer and the quartz wafer are stuck using an inter diffusion bonding method. Primary to the bonding, an etching of both wafers with argon plasma is made. The bonding is realised at low temperature (less than $100^{\circ} \mathrm{C}$ ). We have then made a free quartz membrane that can move. The IDTs have been performed before the bonding step and are located inside the cavity. The resonance frequency of the resonators is designed@433Mhz. Then, a 200 nm thick aluminium layer

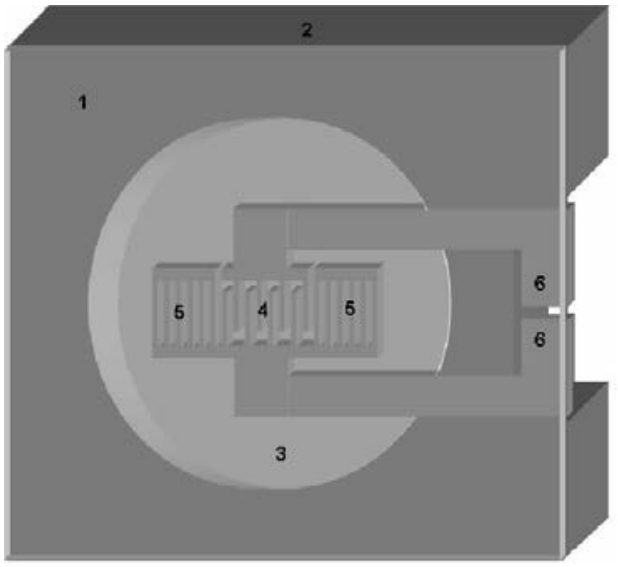

Fig. 1. Schematic 3D view of the sensor.

is evaporated and the IDTs are patterned with the lift-off technique. The thickness of the membrane can be imposed during process to control the frequency shift velocity as a function of pressure. Quartz wafer is thinned with mechanical abrade machine. The thickness of the quartz plate will directly influence the pressure sensitivity of the device. Sensors are then cut in individual parts. Finally, the sensor size is $11 \mathrm{~mm}$ $\mathrm{x} 11 \mathrm{~mm}$ and the diameter of the membrane is $8 \mathrm{~mm}$.

\section{SENSOR MODELING}

The aim of this part is to model the behaviour of surface acoustic waves under stress. In the first part, stress will be considered as homogeneous on each electrode but can vary from one electrode to another. In the second part, the developed model also takes into account the inhomogeneity of the stress along each electrode.

\section{A. Semi-distributed stress modelisation}

1) The P-matrix approach: The approach used in the analysis of SAW devices under stress consists to extract the P-matrix parameters from the results of the infinitely periodic transducer model. The main approximation in the $\mathrm{P}$ matrix model is to consider that it is sufficient, in order to describe a device, to consider two waves propagating in opposite directions. With this assumption, one period of the device can 


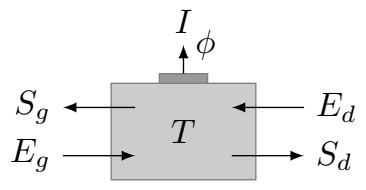

Fig. 2. Base cell of the P-matrix model with two acoustic and one electric ports.

be modeled by a matrix giving the current ant the output wave amplitudes from the voltage on the electrode and the input wave amplitudes. Another assumption is that it is possible to cascade the period, i.e. that there is no wave polarization change from one transducer section to the other and that it is possible to assume that the output wave amplitude for one section is the input wave amplitude for the neighboring section [1].

The quantities $E_{d}$ and $E_{g}$ are incoming amplitude wave, $S_{g}$ and $S_{d}$ are outgoing amplitude waves. $\phi$ is the electrical potential at the base cell and $I$ is the electrical current as we can see on the figure 2. The P-matrix allows to couple electrical and mechanical waves as in equation (1) [3].

$$
\left(\begin{array}{c}
S_{g} \\
S_{d} \\
I
\end{array}\right)=\left[\begin{array}{ccc}
r_{g} & t & \alpha_{g} \\
t & r_{d} & \alpha_{d} \\
-\alpha_{g} & -\alpha_{d} & Y
\end{array}\right]\left(\begin{array}{c}
E_{g} \\
E_{d} \\
\phi
\end{array}\right)
$$

where the value of the coefficient of the P-matrix can be expressed in equation (2).

$$
\begin{aligned}
& t=\cos \Delta \cdot \exp (-j \psi) \\
& r_{g}=-j \sin \Delta \cdot \exp \left[-j\left(\psi+\psi_{r}\right)\right] \\
& r_{g}=-j \sin \Delta \cdot \exp \left[-j\left(\psi-\psi_{r}\right)\right] \\
& Y=G+j B \\
& \alpha_{g}=j \sqrt{G} \cdot \exp \left[-j \frac{\psi+\psi_{r}}{2}\right] \cdot A \\
& \text { with } A=\left[\cos \delta \cdot \exp \left(-j \frac{\Delta}{2}\right)+j \sin \delta \cdot \exp \left(-j \frac{\Delta}{2}\right)\right] \\
& \alpha_{g}=j \sqrt{G} \cdot \exp \left[-j \frac{\psi-\psi_{r}}{2}\right] \cdot B \\
& \text { with } B=\left[\cos \delta \cdot \exp \left(-j \frac{\Delta}{2}\right)-j \sin \delta \cdot \exp \left(-j \frac{\Delta}{2}\right)\right]
\end{aligned}
$$

Where $\sin \Delta$ is the amplitude of the reflection coefficient for one period.

$\psi_{r}$ is the phase between the center of transduction and reflectivity of the cell.

$\delta$ represents the wave directivity.

$G$ is the electromechanical coupling for the wave.

$B$ is the elementary susceptance.

$\psi$ is the phase shift across the cell.

$\delta$ is at first order the phase difference between the transduction center and the reflection center and is null for non directive cut while it is equal to $\pi / 4$ for NSPUDT cuts.

2) The perturbation method: The perturbation method consists in determining the velocity change of surface acoustic waves under stress [2]. Velocity variation with stress can be given by the equation (3).

$$
\left(\frac{\Delta \mathrm{f}}{f_{0}}\right)=\mathrm{S}_{\alpha_{\mathrm{ij}}} T_{\mathrm{ij}}=\mathrm{S}_{\alpha_{11}} T_{11}+\mathrm{S}_{\alpha_{33}} T_{33}
$$

Where $S_{\alpha_{11}}$ and $S_{\alpha_{33}}$ correspond to the linear sensitivity strain coefficients for radial and orthoradial strain seen by Rayleigh waves. These coefficients depends only of the quartz cut choice and are intrinsic of the material. $T_{11}$ and $T_{33}$ are corresponding stress associated to these directions.

3) Stress modeling : We assume for the supposed model that the frequency shift induced with stress is linked to the phase shift across the elementary cell of the resonator as describe in equation (4).

$$
\frac{\partial f}{\partial T}=\frac{\partial \psi}{\partial T}
$$

Where $\mathrm{T}$ represents the stress seen by the resonator i.e. $\psi_{\text {stress }, n}=\psi_{\text {unstress }, n}\left(1+\frac{\Delta f}{f_{0}}\right)$ where $\psi_{\text {stress }, n}$ is linked to the phase shift across the elementary cell number $\mathrm{n}$, $\psi_{\text {unstress, } n}$ corresponds to the phase in the elementary cell number $\mathrm{n}$ for unstress material and $\left(\frac{\Delta \mathrm{f}}{f_{0}}\right)$ represents the relative frequency shift induced by stress seen by the Rayleigh wave for electrode number $\mathrm{n}$ as in equation (3).

The second step consists in computing the P-matrix of the complete device by cascading the P-matrix stress dependancies of the unit cell.

\section{B. Distributed stress modelisation}

Now, we will consider the case where the stress is completely inhomogeneous under the resonator.

1) Stress and sensor: For the calculation, the membrane of the sensor is assumed as an isotropic material. In the case of the developed pressure sensor, the stress seen by the Rayleigh waves is inhomogeneous along each electrode. Locally, tangential and radial stress under the membrane are given by the equation (5) and (6) [4].

$$
\begin{gathered}
\sigma_{r}(p)=\frac{p}{8} \cdot \frac{6}{h^{3}}\left(R^{2} \cdot(1+\nu)-r^{2}(3+\nu)\right) \cdot z \\
\sigma_{\theta}(p)=\frac{p}{8} \cdot \frac{6}{h^{3}}\left(R^{2} \cdot(1+\nu)-r^{2}(1+3 \cdot \nu)\right) \cdot z
\end{gathered}
$$

However, if we consider the infinitesimaly small resonator at the altitude $\mathrm{Z}$ as we can see on the figure 3 , by projected the value of local and tangential stress, the stress seen by the Rayleigh wave is given by the equation (7) and (8).

$$
\begin{gathered}
\sigma_{r}(p, Z)=\frac{p}{8} \cdot \frac{6}{h^{3}}\left(R^{2} \cdot(1+\nu)-r_{e}^{2}(3+\nu)\right) \cdot z \cos (\alpha) \\
\quad-\frac{p}{8} \cdot \frac{6}{h^{3}}\left(R^{2} \cdot(1+\nu)-r_{e}^{2}(1+3 \nu)\right) \cdot z \sin (\alpha) \\
\sigma_{\theta}(p, Z)=\frac{p}{8} \cdot \frac{6}{h^{3}}\left(R^{2} \cdot(1+\nu)-r_{e}^{2}(3+\nu)\right) \cdot z \sin (\alpha) \\
\quad+\frac{p}{8} \cdot \frac{6}{h^{3}}\left(R^{2} \cdot(1+\nu)-r_{e}^{2}(1+3 \nu)\right) \cdot z \cos (\alpha)
\end{gathered}
$$

Where $\alpha$ is the angle between $r_{e}$ and $r$. In these equations, $\cos (\alpha)=\frac{r}{\sqrt{r^{2}+Z^{2}}}$ and $\sin (\alpha)=\frac{Z}{\sqrt{r^{2}+Z^{2}}}$. Moreover the notations $\theta$ and $r$ do not correspond to the radial and the tangential 


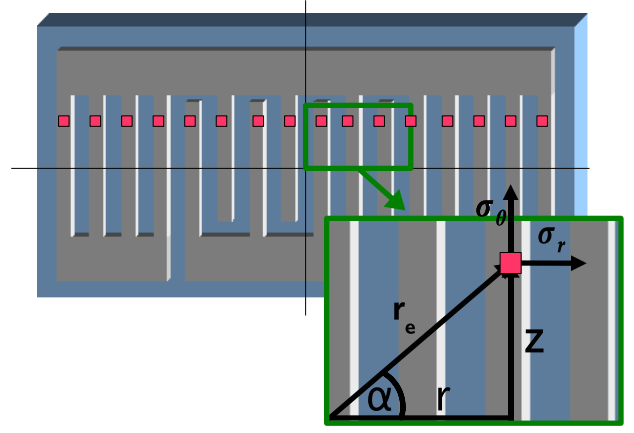

Fig. 3. Schematic view of combination of infinitesimaly resonator used for stress calculation.

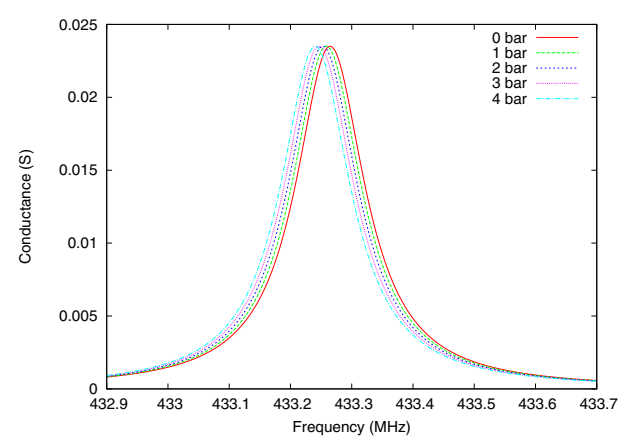

Fig. 4. Evolution of the conductance in the semi-inhomogeneous case.

stress in the membrane as seen previously but to the radial and the tangential stress seen by the Rayleigh waves.

The electrical response of the resonator can be assumed equal to the sum of all infinitesimaly electrical responses of all resonators in parallel.

\section{RESUlts}

In this section, we will show some results of the developed model and we will compare modeling and experimental results.

\section{A. Semi-inhomogeneous case}

Figure 4 show the evolution of the electrical conductance of the developed model in the semi-inhomogeneous case. For the calculation, a $500 \mu \mathrm{m}$ thick membrane has been considered and we assume that stress for each electrode is equal to the stress at the center of the electrode. We can notice that whatever the distributed stress along the resonator, the evolution of the frequency shift induced by the applied pressure is linear. It allows to determine the evolution of the resonance frequency $\omega_{r}(P, Z)$ as in the equation (9).

$$
\omega_{r}(P, Z)=\omega_{r}(0, Z)+f(Z) \cdot P
$$

Where $f(Z)$ corresponds to the slope of the linear response of the infinetisimaly resonator at the altitude $\mathrm{Z}$.

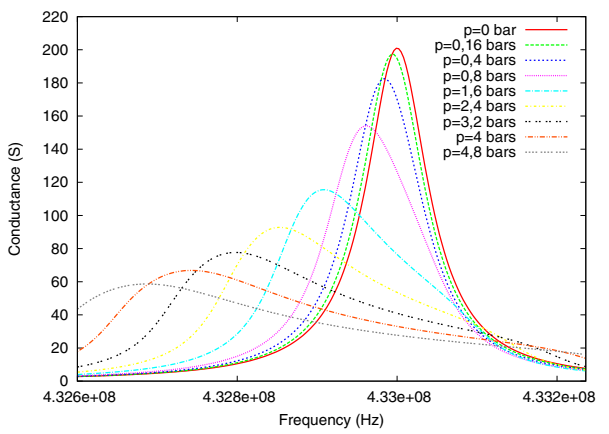

Fig. 5. Evolution of the modeling conductance of the full resonator under inhomogeneous stress.

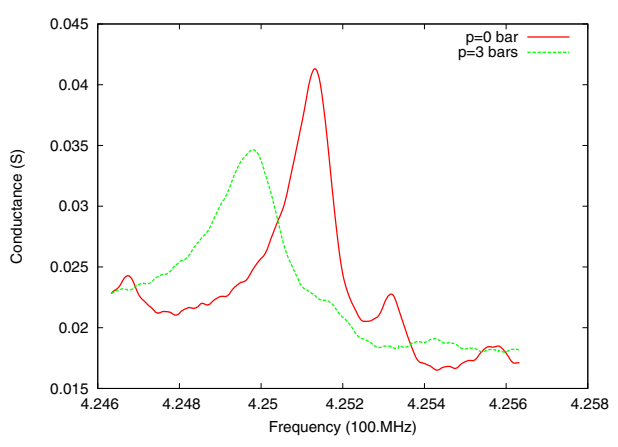

Fig. 6. Evolution of the conductance of the sensor.

\section{B. Inhomogeneous case}

Moreover, figure 4 do not show high variations in the resonance frequency frequency of the resonator. That is why for the calculation of the electrical response for inhomogeneous distributed stress, we will consider that the quality factor of each infinitesimally resonator is constant. By integrating the response of each resonator all along the acoustic aperture of the resonator, we obtain the conductance of the resonator. Figure 5 shows the evolution of the conductance of the resonator for a $150 \mu \mathrm{m}$ thick membrane and a $400 \mu \mathrm{m}$ of acoustic aperture. It shows a fast decrease of the quality factor with the applied pressure. Figure 6 shows experimental datas obtained for the same sensor design as described above.

Moreover, this model shows that the maximum of conductance is not linear with applied pressure. Figure 7 allows to compare theoretical and experimental frequency shift of the sensor with applied pressure.

The figure 9 allows to compare theoretical and experimental evolution of the quality factor with the applied pressure on the sensor. Nevertheless, the developed model can onty shows a decrease of the quality factor under inhomogeneous stress while the experimental measurement shows a low increase of the quality factor for low pressure.

\section{WiRELESS BLOOD PRESSURE MEASUREMENT}

When linked to a dipole antenna, the ssytem allows to realize about ten measurements per second. The graph shows the evolution of the frequency carrier when the sensor is 


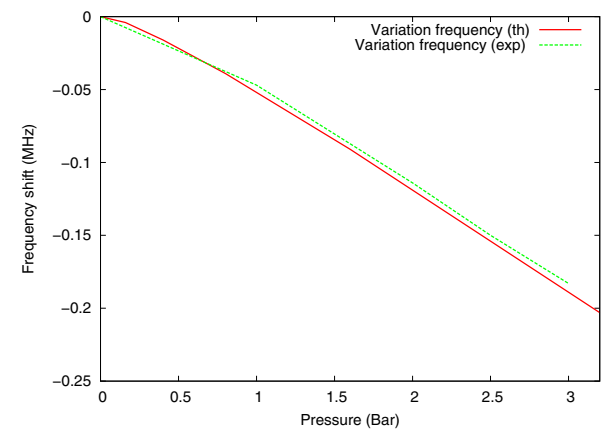

Fig. 7. Comparison of frequency shift expected with developed model and experimental datas.

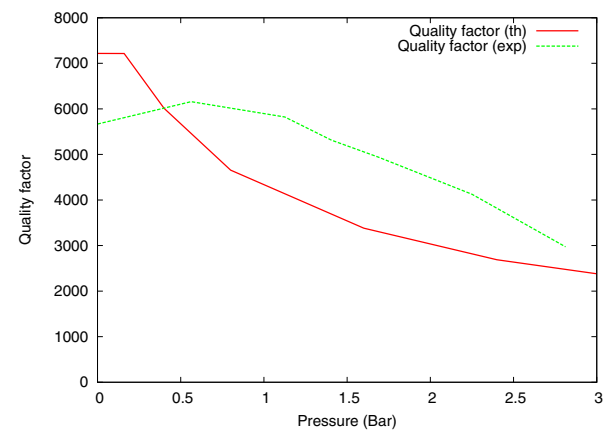

Fig. 8. Comparison of frequency shift expected with developped model and experimental datas.

put between a sygmomanometer and the brachial artery. The length between the antenna and the interrogation unit is about twenty centimeters. The maximum of the carrier amplitude indicates the mean blood pressure.

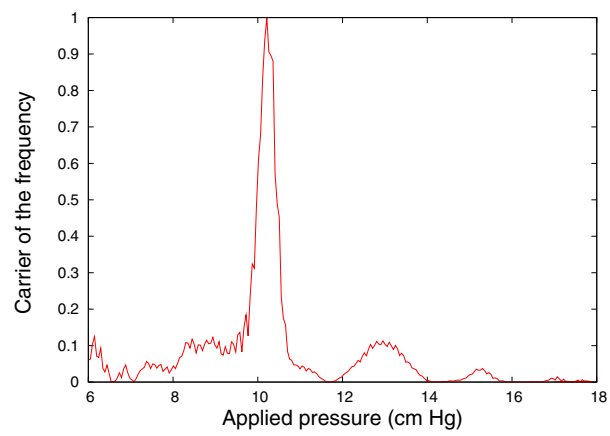

Fig. 9. Evolution of the carrier with applied pressure.

\section{CONCLUSION}

In this paper, a new model for the analysis of inhomogeneous stress in surface acoustic waves has been developed. We have shown that this model allows to determine the frequency shift for the resonance frequency induced by inhomogeneous stress in the resonator. This work enable to otpimize sensor design and future works include a better understanding of the growth of the quality factor for low pressure. Moreover we have shown the possibilty to make wireless measurement.

\section{ACKNOWLEDGMENT}

This material is based on works supported by CIMPA ANR project.

\section{REFERENCES}

[1] S. Ballandras, Precise Modeling of Complex SAW Structures Using a Perturbation Method Hybridized with a Finite Element Analysis. IEEE Trans Ultrason. Symp.,1998.

[2] S. Ballandras, Sensibilité des oscillateurs á quartz á ondes de surfaces aux contraintes et gradients thermiques, 3rd ed. Université de FrancheComté, Besançon, France, 1991.

[3] T. Pastureaud, Etude de nouvelles structures pour la conception et la fabrication de filtres á ondes acoustiques guidées haute frquence. Université de Franche-Comté, Besançon, France, 2002.

[4] S. Timoshenko, Theory of plates and shells. McGraw-Hill, Inc. Second edition, 1980. 